\title{
Deciphering the role of the Bateman domain in IMPDHs
}

B. Raynal ${ }^{1}$, G. Labesse ${ }^{2}$, A. Haouz ${ }^{3}$, T. Alexandre ${ }^{4}$ and $\mathrm{H}$. Munier-Lehmann ${ }^{4}$

${ }^{1}$ Institut Pasteur, Molecular Biophysics Facility, Paris, France; ${ }^{2}$ CNRS UMR5048, Université Montpellier, Centre de Biochimie Structurale, Montpellier, France; ${ }^{3}$ Institut Pasteur, Crystallography Facility, Paris, France; ${ }^{4}$ Institut Pasteur, Chemistry and Biocatalysis Unit, Paris, France.

Bateman domains are regulatory domains of a wide range of proteins. Mutations within these domains are associated with hereditary diseases highlighting their key roles in different cellular functions. The first structure of a Bateman domain comes from the crystal structure of the Chinese hamster inosine-5'-monophosphate dehydrogenase (IMPDH). However the role of this domain at the molecular level was only recently deciphered in IMPDHs thanks to our multidisciplinary approach. IMPDH is an ubiquitous and essential enzyme for de novo guanine nucleotide biosynthesis. Being an important regulator of cell proliferation, human IMPDH has been explored as a target for drug development.

Almost all IMPDHs are composed of two structural domains, namely a catalytic and a Bateman domain. Our characterization of ten different IMPDHs shed light on the functional and structural role of the Bateman domain in IMPDH regulation: the Bateman domain is involved in MgATP-dependent allosteric and oligomeric state regulations. Indeed MgATP is a common effector, which binds to the Bateman domain, and leads to drastic structural rearrangements. According to the effect of this binding on the structural properties (but also on the kinetic behavior), two IMPDH classes were defined. Consequently, we have proposed a model to depict the modulation of the quaternary structure for both classes. Our ongoing work (unpublished data) on different mutants might suggest that the Bateman domain would be necessary and sufficient to confer these structural properties. 